American Journal of Infectious Diseases 8 (1): 34-40, 2012

ISSN 1553-6203

(C) 2012 Science Publications

\title{
Assessment of Cardio-Metabolic Risk Factors among Young Adult Females
}

\author{
Swati Dhruv, Uma Iyer and Kusum Bhatt \\ Department of Foods and Nutrition, \\ Faculty of Family and Community Sciences, \\ WHO Collaborating Centre for Research and \\ Training in Promoting Nutrition in Health and Development, \\ the M S University of Baroda, Vadodara, Gujarat, India
}

\begin{abstract}
Problem statement: Over the past two decades there has been a striking increase in the number of people with metabolic syndrome in developing countries. The current study was thus undertaken to map the prevalence of Metabolic Syndrome (MS) and to assess the cardio-metabolic risk factors among young adult females $(n=1303)$ aged 18-26y from four girls hostel of the Maharaja Sayajirao University of Baroda. Approach: The anthropometric analysis showed a high prevalence of overweight/obesity (20.8\%), abdominal obesity (12.7\%) among the subjects. The clinical profile revealed that $12.1 \%$ were hypertensives. The prevalence of dyslipidemia revealed that no one had hypercholesterolemia and $4.1 \%$ had hypertriglyceridemia, $12.1 \%$ had elevated LDL-C and $40.3 \%$ had low levels of HDL-C. The prevalence of metabolic syndrome was 2.4 and $4.1 \%$ according to the International Diabetes Federation (IDF) and World Health Organization (WHO) criteria respectively. Lipid profile in relation to metabolic syndrome showed that VLDL-C and Triglyceride (TG) values were non-significantly higher among the young adult females and HDL-C values were significantly $(\mathrm{p}<0.001)$ lower among those subjects who had metabolic syndrome. The mean C-Reactive Protein (CRP) level of the study subjects was found to be in normal range, except for $10.5 \%$ who had elevated CRP levels. A high prevalence of multiple risk factors seen among the subjects like low fruits and vegetable consumption, sedentary lifestyle, hypertension, obesity. Results: The three common and predominant risk factors $(>80 \%)$ identified were lower intake of fruits $(81.5 \%)$, vegetables $(96 \%)$ and physical inactivity $(88.7 \%)$. The other risk factors which were present between $30-50 \%$ were hypertension, lower HDL-C, Body Mass Index (BMI) and Waist Circumference (WC). Among the non-modifiable factor heredity component was present in $34 \%$ of the subjects. Conclusion: The study highlights that lifestyle factors had equivalent risk for overweight and metabolic syndrome. Multiple risk factor scenario calls for lifestyle management to avert later consequences.
\end{abstract}

Key words: Metabolic syndrome, cardio-metabolic, International Diabetes Federation (IDF), Body Mass Index (BMI), Systolic Blood Pressure (SBP), Diastolic Blood Pressure (DBP)

\section{INTRODUCTION}

The MS is a cluster of cardiovascular and diabetic risk factors. It is estimated that around $20-25 \%$ of the world's adult population (Mangat et al., 2010). Persons with the metabolic syndrome are at essentially twice the risk for cardiovascular disease compared with those without the syndrome. It further raises the risk for type 2 diabetes by about 5 -fold (Grundy, 2008).

The clinical manifestations of MS vary in different populations. Caucasians mainly show dyslipidemia, African populations show hypertension, Native Americans show hyperglycemia and South Asians show both hyperglycemia and accelerated CHD (Bloomgarden, 2000).

The prevalence of MS and CVD among South Asians is increasing. This trend is not only seen in South Asians residing on the Indian subcontinent (e.g., India, Pakistan, Bangladesh, Nepal), but is also observed in countries with large numbers of South Asian immigrants. It is estimated that $20-25 \%$ of South Asians have developed MS and many more may be

Corresponding Author: Swati Dhruv, Department of Foods and Nutrition, Faculty of Family and Community Sciences, WHO Collaborating Centre for Research and Training in Promoting Nutrition in Health and Development, The M S University of Baroda, Vadodara 390 002, Gujarat, India Tel: 09898078988 
prone to it (Eapen et al., 2009). Urbanization, economic growth, irregular timing of meals and dietary westernization has been suggested as potential culprits implicated in the development of this disorder.

India is in a state of nutrition transition with advancement of technological and agriculture evolution. This has led to changes in lifestyle and dietary practices. The clustering of risk factors leading to the development of MS has increased over the past two decades, including developing countries. Hence, keeping in mind the various modifiable and non modifiable risk factors leading to development of MS and its co-morbidities, the present study was planned to address this issue among young adult's females. The age group was focused because it represents a graduation from adolescence to adult stage that involves several physical as well as environmental changes and moreover studies have shown that the prevalence of obesity is higher in females than males.

\section{MATERIALS AND METHODS}

Study population: The study population comprised of young adult females residing in 4 hostels of the Maharaja Sayajirao University of Baroda $(n=1415)$, out of which 1303 could be contacted in 3 visits.

Measurement: On 1303 girls the following information was elicited which is given below.

Biochemical measurement: Based on the results of anthropometric measurement from 1st phase all the enrolled subjects were classified based on Asia Pacific Classification as Underweight, Normal, Overweight and Obese. The other variable that was included was the presence of hypertension as the overall prevalence of hypertension was $12.6 \%$ based on BP measurement. Thus a $2 * 2$ factorial design was chosen by taking into consideration the BMI and BP measurement. Random selection was done for biochemical estimation based on the willingness to participate in the study. Around 124 girls were randomly selected for biochemical estimation. All the variables including Fasting Blood Glucose (FBG), Total Cholesterol (TC), TG, LDL-C and HDL-C were measured using enzymatic methods in a reputed pathology laboratory of Vadodara.

The study was approved by the institution medical ethics committee (No. FCSC/FND/ME/41).

Statistical analysis: Results are expressed as Mean \pm $\mathrm{SD}$ and as percentages. The significance of the data was evaluated using ANOVA and students ' $t$ ' test. Correlation test was used to find correlation between the two variables. Relative risk was used to find \% high risk of disease and Chi square was done to calculate association between the two variables. All tests were considered significant at $p<0.05$ level. The statistical analysis was carried out using Microsoft Excel.

\section{RESULTS}

The clinical profile of the subjects showed that the age of the subjects ranged from 18-26 years. Predominantly 53.2 and $21.8 \%$ of the subjects were in $19-21 \mathrm{y}$ and $22-24 \mathrm{y}$ age respectively and the mean age of the subjects was 21 y having a mean BMI of 20.5 and WC $69 \mathrm{~cm}$, WHR 0.7 and the mean WSR was in the normal range $(<0.5)$. The mean $\mathrm{BP}$ measurements were normal (Table 1).

According to the Asia Pacific criteria the prevalence of overweight and obesity was found to be 9.0 and $12.6 \%$ respectively. The WHO classification underestimated the prevalence and it was 10.1 and $2.5 \%$ respectively for overweight and obesity. The prevalence of underweight was very high and nearly $1 / 3 \mathrm{rd}(33 \%)$ of the population were underweight. This observation highlights the dual burden of malnutrition among young hostel girls (Table 2).

The abdominal obesity was assessed using the 3 anthropometric indices-WC, WHR and WSR. By all the 3 indices, the percent prevalence of abdominal obesity ranged from $12.7-21.1 \%$, the least $(12.7 \%)$ being with IDF criteria and highest with WHR criteria (21.1\%) (Table 3).

The information on anthropometric measurement in relation to age of menarche is given in Table 4 The prevalence of overweight/obesity and abdominal obesity declined as the age of menarche increased, thereby indicating that early menarche had a significant influence on the prevalence of overweight/obesity $\left(\mathrm{x}^{2}=31.1, \quad \mathrm{p}<0.001\right)$ and abdominal obesity $\left(\mathrm{x}^{2}=37.8, \mathrm{p}<0.001\right)$.

Over all prevalence of hypertension was $12.6 \%$, out of this $9.3 \%$ had elevated SBP and $12.8 \%$ had elevated DBP. Predominantly the subjects were in prehypertensive stage.

A sizeable portion of the study group (86.5\%) reported sedentary life style (No regular physical activity or $<3$ hours/week). Among the subjects who exercised nearly $60.2 \%$ of the subjects did exercise on a regular basis and it was $>3 \mathrm{hrs} /$ week. 
Am. J. Infect. Dis., 8 (1): 34-40, 2012

Table 1: Variables under study

\begin{tabular}{lll}
\hline Variables & Parameters assessed & Tools \\
\hline Anthropometry & Height, weight, BMI, WC, Hip Circumference, & Bathroom scale and fiber glass tape \\
& Waist Hip Ratio (WHR), Waist-to-Stature Ratio (WSR), & \\
neck, wrist, forearm circumference, & Pretested questionnaire \\
Socioeconomic Status & Religion, marital status, education & Standard procedures \\
Medical History & CHD, diabetes, obesity, hypertension, thyroid & Sphygmomanometer \\
Blood Pressure (BP) & Systolic Blood Pressure (SBP), Diastolic Blood & Food frequency questionnaire \\
Dietary Information & Pressure (DBP) & \\
& Type of diet, frequency consumption of breakfast, \\
& lunch, fruits and vegetables, soft drinks, junk foods, \\
frequency of consuming outside & \\
\hline
\end{tabular}

Table 2: Clinical information of young adult female subjects (Mean $\pm \mathrm{SD}$ )

\begin{tabular}{ll}
\hline Variable & Values \\
\hline Number of subjects & 1303 \\
Age $(\mathrm{y})$ & $20.3 \pm 2.22$ \\
Height $(\mathrm{cm})$ & $157 \pm 6.14$ \\
Body Weight $(\mathrm{kg})$ & $50.6 \pm 10.02$ \\
BMI $\left(\mathrm{kg} \mathrm{m}^{-2}\right)$ & $20.5 \pm 3.76$ \\
Waist circumference $(\mathrm{cm})$ & $68.9 \pm 8.73$ \\
Hip $(\mathrm{cm})$ & $91.2 \pm 8.60$ \\
WHR & $0.7 \pm 0.05$ \\
WSR & $0.44 \pm 0.06$ \\
Neck circumference $(\mathrm{cm})$ & $30.1 \pm 1.73$ \\
Wrist $(\mathrm{cm})$ & $14.3 \pm 0.89$ \\
Forearm $(\mathrm{cm})$ & $21.6 \pm 1.56$ \\
Diastolic blood pressure $(\mathrm{mmHg})$ & $71.9 \pm 8.16$ \\
Systolic blood pressure $(\mathrm{mmHg})$ & $108.6 \pm 10.15$ \\
Menarche age $(\mathrm{Y})$ & $13.3 \pm 1.59$ \\
\hline
\end{tabular}

Table 3: Prevalence of obesity among young adult female subjects

\begin{tabular}{lll}
\hline Classification & Asia pacific & WHO \\
\hline Normal & $592(45.4)$ & $709(54.4)$ \\
Underweight & $430(33.0)$ & $430(33.0)$ \\
Overweight & $117(9.0)$ & $131(10.1)$ \\
Obesity & $164(12.6)$ & $33(2.5)$ \\
Grade I & $131(10.1)$ & $31(2.4)$ \\
Grade II & $33(2.5)$ & $2(0.2)$ \\
\hline
\end{tabular}

Value in parentheses indicate percentages

Table 4: Prevalence of abdominal obesity among young adult female subjects

\begin{tabular}{ll}
\hline Parameter & Females, $\mathrm{n}(\%)$ \\
\hline Waist Circumference (WC) $\geq 80 \mathrm{~cm}$ cut-off & $165(12.7)$ \\
Waist Hip Ratio (WHR) $\geq 0.8$ cut-off & $275(21.1)$ \\
Waist Stature Ratio (WSR) $\geq 0.5$ cut-off & $209(16.1)$ \\
\hline
\end{tabular}

Dietary habits: Majority of the subjects was vegetarians $(76.2 \%)$ and nearly $67 \%$ of the subjects had breakfast regularly, which is a good habit and $28 \%$ of the subjects' skipped lunch. On an average $89 \%$ of the subjects was consuming outside food at some or other time, out of which $7.8 \%$ were having outside food on daily basis and $50 \%$ on weekly basis. The consumption pattern of fruits and Green Leafy Vegetables (GLVs) was very poor. Only 18.9 and $2.2 \%$ girls were taking fruits and GLVs >3days in a week respectively and 43.4 and $44.6 \%$ were not having fruits and vegetable even once a week. Consumption of milk and milk products was low, with $26.2,33.9$ and $49.2 \%$ having milk, curd and buttermilk respectively.
Table 5: Prevalence of overweight, obesity and abdominal obesity in relation to menarche age $(\%)$

\begin{tabular}{lllll}
\hline Variables & $8-10 \mathrm{Y}$ & $11-12 \mathrm{Y}$ & $>12 \mathrm{Y}$ & $\mathrm{x}^{2}$ \\
\hline Overweight & $16.6(4)$ & $12.1(36)$ & $6.8(67)$ & $31.1^{* * *}$ \\
Obese & $29.1(7)$ & $17.8(53)$ & $10.6(104)$ & \\
WC $>80 \mathrm{~cm}$ & $41.6(10)$ & $19.5(58)$ & $9.9(97)$ & $37.8^{* * *}$ \\
\hline ***; Significant at $\mathrm{p}<0.001$ & & &
\end{tabular}

The data on food consumption pattern based on BMI revealed that nearly $40 \%$ obese and $38 \%$ of overweight subjects skipped lunch as against $25 \%$ for normal and underweight subjects. Similarly breakfast skipping was higher in overweight/obese subjects (26$29 \%)$ than underweight and normal subjects (16\%).

Biochemical estimation: With regard to the biochemical parameters, the mean FBG value was 78 $\mathrm{mg} \mathrm{dL}{ }^{-1}$, which falls in the normal range indicating that none of the subjects were diabetics (Table 5). The mean value for TC, TG and HDL-C was 138, 89 and $41 \mathrm{mg}$ $\mathrm{dL}^{-1}$ respectively. The mean value for atherogenic lipoprotein fraction i.e., LDL-C and VLDL-C was 80 and $18 \mathrm{mg} \mathrm{dL}^{-1}$ respectively. The mean atherogenic indices $\mathrm{TG} / \mathrm{H}, \mathrm{TC} / \mathrm{H}, \mathrm{L} / \mathrm{H}$ and $\mathrm{TC} / \mathrm{L}$ ratio were normal and were 3, 4, 2 and 2 respectively. The mean CRP values were $0.4 \pm 0.2$ (Table 5) and $10.5 \%$ girls had high CRP value (>0.6 mg. dl).

The prevalence of dyslipidemia showed that no one had hypercholesterolemia, $4.1 \%$ had hypertriglyceridemia, $12.1 \%$ had elevated levels of atherogenic lipoprotein LDL-C and $40.3 \%$ had low HDL-C levels ( $\left.<40 \mathrm{mg} \mathrm{dL}^{-1}\right)$. The overall prevalence of metabolic syndrome was $2.4 \%$ and $4.1 \%$ according to IDF and WHO respectively (Table 6). Lipid profile was studied in relation to various variables, anthropometric measurements and physical activity, education, type of diet and presence and absence of metabolic syndrome; however no significant differences were obtained.

Many risk factors were found to be present among the young adult females and an overview of risk factors presented in Fig. 1. A range of 11 risk factors have been depicted in the graph. The graph clearly indicates that more than $30 \%$ of the subjects had one or more risk factors, indicating multiple risk factor scenarios. 
Am. J. Infect. Dis., 8 (1): 34-40, 2012

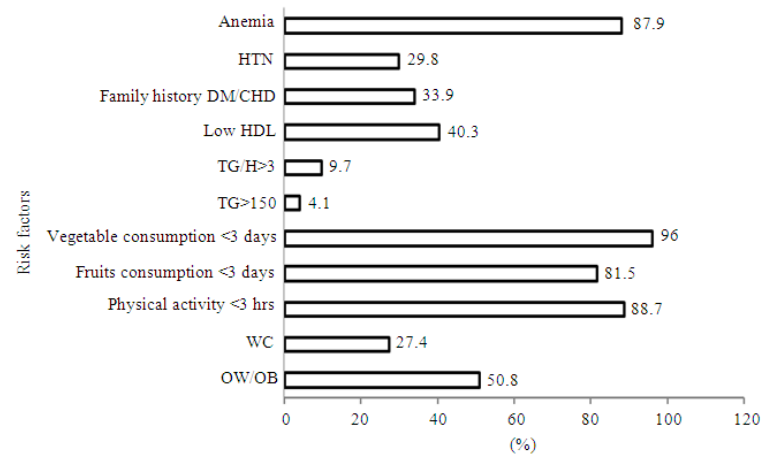

Fig. 1: Risk factor scenario among young adult female subjects

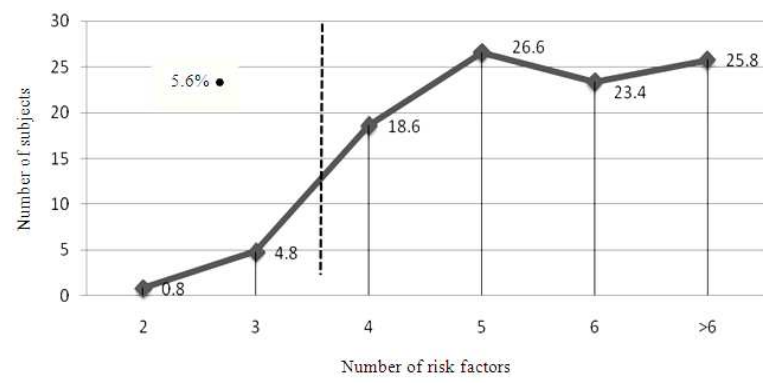

Fig. 2: Frequency distribution of subjects based on number of risk factors

Table 6: FBG, lipid profile and CRP values of young adult female subjects (Mean $\pm \mathrm{SD}, \mathrm{mg} \mathrm{dL}^{-1}$ )

\begin{tabular}{ll}
\hline Variable & Value \\
\hline $\mathrm{N}$ & 124 \\
FBG & $78 \pm 6.1$ \\
TC & $138 \pm 20.1$ \\
HDL-C & $41 \pm 2.9$ \\
LDL-C & $80 \pm 16.5$ \\
VLDL-C & $18 \pm 5.7$ \\
TG & $89 \pm 28.2$ \\
TC/H & $4 \pm 0.40$ \\
L/H & $2 \pm 0.4$ \\
TC/L & $2 \pm 0.2$ \\
TG/H & $3 \pm 0.7$ \\
CRP & $0.4 \pm 0.2$ \\
\hline
\end{tabular}

Table 7: Prevalence of Metabolic syndrome among young adult female subjects $(\%)$

\begin{tabular}{ll}
\hline $\mathrm{IDF}(2006)$ & WHO (1995) \\
\hline 2.4 & 4.1 \\
\hline
\end{tabular}

The 3 common and predominant risk factors $(>80 \%)$ identified were low intake of fruits $(81.5 \%)$, vegetables $(96 \%)$ and physical inactivity $(88.7 \%)$. The other risk factors which were present between $30-50 \%$ were hypertension, low HDL-C, BMI and WC. Among the non modifiable factor heredity component was present in $34 \%$ of the subjects. When the frequency distribution of subjects according to the number of risk factors present (Fig. 2) was done an alarming $25.8 \%$ of the subjects showed $>6$ risk factors and $23.4 \%$ had 6 risk factors. About 26.6 and $18.6 \%$ had 5 and 4 risk factors which are also regarded as high. Only 4.8 and $0.8 \%$ of the subjects had 3 and 2 risk factors respectively.

Overall the prevalence of Iron Deficiency Anemia (IDA) was found to be $87.9 \%$. The prevalence of anemia ranged from $81-95 \%$ and was found to be max in underweight girls $(95.15 \%)$.

\section{DISCUSSION}

In the present study, the prevalence of metabolic syndrome was found to be 2.4 and $4.1 \%$ according to IDF and WHO criteria respectively (Table 7). This lower prevalence of MS could be attributed to the younger age of the subjects. The Third National Health and Nutrition Examination Survey among US adults showed that the prevalence increased from $6.7 \%$ among participants aged 20 through 29 years to 43.5 and $42.0 \%$ for participants aged 60 through 69 years and aged at least 70 years respectively (Ford et al., 2002), thus indicating that advancing age would be a predisposing factor for MS and that preventive measures need to be taken at the younger age.

In the present study, dual burden of malnutrition was witnessed in young adult female subjects. The gravity of the problem was more for undernutrition (33\%) than overnutrition (20.8\%), though both the spectrum has adverse consequences later in life. In the present study one fifth of the study population fell under the category of overnutrition. This could be due to the fact that majority of girls were not involved in any kind of physical activity and also had faulty dietary habits (high calorie, fat and low fruits and vegetables).

The overall prevalence of anemia was $87.9 \%$, which could be attributed to the low intake of iron rich foods and poor iron bioavailability. This is an important finding which reveals that Iron deficiency anemia is also widely prevalent among the middle and upper income adult female subjects.

The prevalence of abdominal obesity in the study subjects was $12.7 \%$ according to $\mathrm{WC}$ and $21.1 \%$ by to WHR criteria, thereby indicating that with advancing age the risk of developing insulin resistance and its consequences is very high. Similar findings have been reported by other researchers who have shown that shown that visceral fat correlates with health risks to a greater extent than does adipose tissue in other regions of the body (Wang and Hoy, 2004; Zhu et al., 2002). 
The data also revealed that girls who had early menarche had significantly higher BMI. These results are in line with studies that have shown that early menarche is characterized by excess body fatness and insulin beginning in early childhood and higher prevalence of clustering of adverse levels of risk variables of metabolic Syndrome $\mathrm{X}$ in young adulthood (Frontini et al., 2003).

As a faulty lifestyle would predispose an individual to various alterations in lipid profile, some of the general lifestyle related factors of the subjects were studied. In relation to physical activity it was found that $86.5 \%$ of the subjects did not exercise at all. Among the subjects who exercised, no appreciable changes in the lipid profile was observed in comparison to those who did not exercise. However it was found that among the subjects who exercised the TC component showed a non significant reduction. This non significant reduction between exercising and non-exercising subjects could be due to the irregularity in the exercise schedule.

The growing prevalence of obesity is increasingly recognized as one of the most important risk factors for the development of hypertension. In present study the prevalence of hypertension was $12.6 \%$ and it shows that hypertension was significantly $(\mathrm{p}<0.001)$ higher among overweight and obese girls and it was found to be 6 times higher in obese than in normal girls. Based on population studies, risk estimates indicate that at least two-thirds of the prevalence of hypertension can be directly attributed to obesity (Krause et al., 1998; Iyer et al., 2010). These results indicate that these subjects would be at a higher risk to suffer from DM and CVD at a later stage with advancing age. The same is substantiated by a study carried out by Narkiewicz (2006) that the epidemic of obesity and obesity-related hypertension is paralleled by an alarming increase in the incidence of diabetes mellitus and chronic kidney disease.

In the present study, the contributions to hyperlipidemia (present in $<10 \%$ subjects) were low level of HDL-C, high levels of TG and LDL-C levels. Further the lipid levels were not influenced by a number of variables, thereby indicating the protective effect at younger age. Similar findings have been shown by Gupta et al. (2009).

The mean CRP level of the study subjects was found to be in normal range $\left(0.4 \mathrm{mg} \mathrm{dL}^{-1}\right)$. This might also be due to early age of the participants. However studies have shown that measurement of CRP adds clinically important prognostic information to the metabolic syndrome (Nazmi et al., 2008; Vikram et al., 2003; Ridkel et al., 2003).
Risk factor analysis: Although aberration in lipid profile was not observed in current study population there was high prevalence of multiple risk factors seen among the subjects like low fruits and vegetable consumption, sedentary lifestyle, hypertension, obesity.

The 3 common and predominant risk factors (>80\%) identified were low intake of fruits $(81.5 \%)$, vegetables (96\%) and physical inactivity (88.7\%). These results are in line with the study carried out by by Iyer et al. (2011). Another study carried out among the Australian-Lebanese population also revealed physical inactvity as one of the cardiovascular risk factors (Shahwan-Akl, 2010). The other risk factors which were present between 30-50\% were hypertension, low HDL-C, BMI and WC. Among the non-modifiable factor heredity component was present in $34 \%$ of the subjects. Gupta et al. (2009) have also reported similar findings in young adult population, where by low prevalence of multiple cardiovascular risk factors in adolescents and rapid escalation of these risk factors by age of 30-39 years was noted in urban Asian Indians.

When the frequency distribution of subjects according to number of risk factors present was done, an alarming $25.8 \%$ of the subjects showed $>6$ risk factors and $23.4 \%$ had 6 risk factors. If uncontrolled, these risk factors may further bring about aberration in lipid profile, thereby predisposing the subjects to towards diabetes and CVD. The World Health Report, 2002 has identified 8 risk factors which can be easily measured in populations that contribute the most to mortality and morbidity, but which can be reduced through primary intervention.

Cardiovascular diseases in developing countries are characterized by early age of onset and greater mortality (Beaglehole and Yach, 2003). Worldwide data reveal that while more than $70 \%$ of deaths in high income countries occur after the age of 70 years, in low and middle income countries more than $70 \%$ deaths occur below this age (Reddy, 2004). Coronary heart disease occurs at least ten years earlier in South Asians as compared to other ethnic groups and the average age of stroke is much lower than in the western countries. The inter heart study reported that multiple cardiovascular risks occurred at a younger age among South Asians which explained younger age of acute myocardial infarction in these subjects which can be largely explained by higher risk factor levels at younger ages and the cardiovascular risk factors important in older subjects were equally important in the young South Asians (Joshi et al., 2007; Yusuf et al., 2004). The present study also shows a younger age of 
escalation of multiple cardiovascular risk factors which is in line with a study that has reported a moderate to high prevalence of multiple atherosclerosis risk factors in the adolescents and the young (Vikram et al., 2003).

From the above mentioned results it is thus recommended to arrest the progression of these multiple risk factors of MS at an early age so as to get avoid complications related to it at a later stage in their life. Thus primordial preventive strategies can be planned for reducing the risk of NCDs in this young population.

\section{CONCLUSION}

Going by the observation of the present study we strongly feel that monitoring of risk factors at an early age would go a long way in reducing the burden of NCDs. Secondly people should be encouraged to improve their lifestyle habits (Physical activity, Diet) for weight management and improving insulin sensitivity. Simple messages addressing these two issues would help in alleviating NCDs in India.

\section{REFERENCES}

Beaglehole, R. and D. Yach, 2003. Globalisation and the prevention and control of non-communicable disease: The neglected chronic diseases of adults. Lancet, 362: 903-908. DOI: 10.1016/S01406736(03)14335-8

Bloomgarden, Z.T., 2000. Obesity and diabetes. Diab. Care., 23: 1584-1590. DOI: 10.2337/diacare.23.10.1584

Eapen, D., G.L. Kalra, N. Merchant, A. Arora and B.V. Khan, 2009. Metabolic syndrome and cardiovascular disease in South Asians. J. Vascular Health Risk Manage., 5: 731-743. DOI: 10.2147/VHRM.S5172

Ford, E.S., W.H. Giles and W.H. Dietz, 2002. Prevalence of the metabolic syndrome among us adults. JAMA, 287: 356-359. DOI: 10.1001/jama.287.3.356

Frontini, M.G., S.R. Srinivasan and G.S. Berenson, 2003. Longitudinal changes in risk variables underlying metabolic Syndrome $\mathrm{X}$ from childhood to young adulthood in female subjects with a history of early menarche: The bogalusa heart study. Int. J. Obes. Relat. Metab. Disord., 27: 1398-1404. PMID: 14574352

Grundy, S.M., 2008. Metabolic syndrome pandemic. Arterioscler Thromb Vasc Biol, 28: 629-636. DOI: 10.1161/ATVBAHA.107.151092
Gupta, R., A. Misra, N.K. Vikram, D. Kondal and S.S. Gupta et al., 2009. Younger age of escalation of cardiovascular risk factors in Asian Indian subjects. BMC Cardiovasc. Disord., 9: 28-28. DOI: 10.1186/1471-2261-9-28

Iyer, U., G. Mathur, N. Panchanmiya and S. Dhruv, 2011. Risk factor scenario in an industrial set-up: Need for an effective screening tool to assess the high-risk group. Indian J. Community Med., 35: 262-266. DOI: 10.4103/0970-0218.66884

Iyer, U., P. Desai, S. Dhruv and V. Venugopal, 2010. Risk factor analysis for cardiovascular diseases in pregeriatric type 2 diabetes mellitus subjects. Int. J. Contemporary Res. Rev.

Joshi, P., S. Islam, P. Pais, S. Reddy and P. Dorairaj et al., 2007. Risk factors for early myocardial infarction in South Asians compared with individuals in other countries. JAMA, 297: 286294. DOI: 10.1001/jama.297.3.286

Krause, R.M., M. Winston, B.J. Fletcher and S.M. Grundy, 1998. Obesity: Impact on cardiovascular disease. Circulation, 98: 1472-1476. DOI: 10.1161/01.CIR.98.14.1472

Mangat, C., N.K. Goel, D.K. Walia, N. Agarwal and M.K. Sharma et al., 2010. Metabolic syndrome: A challenging health Issue in highly urbanized Union Territory of north India. Diabetol. Metab. Syndrome, 2: 19-19. DOI: 10.1186/1758-5996-219

Narkiewicz, K., 2006. Obesity and hypertension-the issue is more complex than we thought. Nephrol. Dialysis Trans., 21: 264-267. DOI: 10.1093/ndt/gfi290

Nazmi, A., D.C. Gonzalez, I.O. Oliveira, B.L. Horta and D.P. Gigante et al., 2008. Life course weight gain and C-reactive protein levels in young adults: Findings from a Brazilian birth cohort. Am. J. Hum. Biol., 21: 192-199. PMID: 19107921

Reddy, K.S., 2004. Cardiovascular diseases in nonwestern countries. Eng. J. Med., 350: 2438-2440. DOI: 10.1056/NEJMoa1106920

Ridkel, P.M., J.E. Buring, N.R. Cook and N. Rifai, 2003. C-reactive protein, the metabolic syndrome and risk of incident cardiovascular events: An 8Year Follow-Up of 14719 initially healthy american women. Circulation, 107: 391-397. DOI: 10.1161/01.CIR.0000055014.62083.05

Shahwan-Akl, L., 2010. Cardiovascular disease risk factors among adult australian-lebanese in melbourne. Int. J. Res. Nurs., 1: 1-7. DOI: 10.3844/ijrnsp.2010.1.7 
Vikram, N.K., A. Misra, M. Dwivedi, R. Sharma and R.M. Pandey et al., 2003. Correlations of Creactive protein levels with anthropometric profile, percentage of body fat and lipids in healthy adolescents and young adults in urban North India. Atherosclerosis, 168: 305-313. DOI: 10.1016/S0021-9150(03)00096-0

Wang, Z. and W.E. Hoy, 2004. Waist circumference, body mass index, hip circumference and waist-tohip ratio as predictors of cardiovascular disease in Aboriginal people. Eur. J. Clin. Nutr., 58: 888-893. PMID: 15164109
Yusuf, S., S. Hawken, S. Ounpuu, T. Dans and A. Avezum et al., 2004. Effect of potentially modifiable risk factors associated with myocardial infarction in 52 countries (the inter heart study): Case control study. Lancet, 364: 937-52. DOI: 10.1016/S0140-6736(04)17018-9

Zhu, S., S. Heshka, Z.M. Wang and S.B. Heymsfield, 2002. A combined measure of BMI and Waist Circumference (WC), compared to BMI. FASEB J., 16: 612. 